\title{
NUEVO RÉCORD DE TRAMETES HIRSUTA (WULFEN) LLOYD (BASIDIOMYCOTA) EN REFUGIO DE VIDA SILVESTRE LAGUNA MALLÉN, SAN PEDRO DE MACORÍS-REPÚBLICA DOMINICANA
}

\section{New record of Trametes hirsuta (Wulfen) Lloyd (Basidiomycota) at Laguna Mallén Wildlife Refuge, San Pedro de Macorís-Dominican Republic}

\section{Mayeli Jaime De Aza}

Instituto Superior de Formación Docente Salomé Ureña (ISFODOSU), Santo Domingo, República Dominicana. ORCID: 0000-0002-3345-2113

Correo-e: mayeli.jaime@isfodosu.edu.do

\section{José Guillermo de la Rosa}

Grupo de Investigación Biodiversidad, Ecología y Conservación (Universidad ISA), Santiago, República

Dominicana. ORCID: 0000-0003-2257-1528

Correo-e: lic.joseguillermodelarosa@gmail.com
Héctor Julio Santos Báez

Ministerio de Educación de la República Dominicana (MINERD), Santo Domingo, República Dominicana. ORCID: 0000-0002-6448-808X

Correo-e: hectorjuliosantosbaez@gmail.com

Recibido: 13/5/2021 • Aprobado: 21/6/2021

Cómo citar: Jaime De Aza, M., de la Rosa, J. G., \& Santos Báez, H. J. (2021). Nuevo récord de Trametes hirsuta (Wulfen) Lloyd (Basidiomycota) en Refugio de Vida Silvestre Laguna Mallén, San Pedro de Macorís-República Dominicana. Ciencia, Ambiente Y Clima, 4(1), 19-27. https://doi.org/10.22206/cac.2021.v4i1.pp19-27

\section{Resumen}

Se reporta por primera vez la especie Trametes hirsuta sobre tronco de Hura crepitans L., en el Refugio de Vida Silvestre Laguna Mallén. T. hirsuta es un hongo fitopatógeno del orden de los polyporales que causa pudrición blanca en madera. Su carpóforo es de color blanquecino a pardoamarillento, con un margen de tonalidad marrón. Son productores de lacasas y degradadores de lignina. Se asientan sobre madera de angiospermas.

Palabras clave: hongo fitopatógeno; orden polyporales; organismos saprófitos; podredumbre blanca; primer reporte.

\begin{abstract}
The species Trametes hirsuta is reported for the first time on trunks of Hura crepitans L., in the Laguna Mallén Wildlife Refuge. T. hirsuta is a phytopathogenic fungus of the order Polyporales that causes white rot in wood. Its carpophore is whitish to yellowish-brown with a brownish margin. They are laccase producers and lignin degraders. They settle on angiosperm wood.
\end{abstract}

Keywords: Phytopathogenic fungus; Order polyporales; Saprophytic organisms; White rot; First report. 


\section{Introducción}

En los últimos años el mundo de los hongos se ha convertido en un amplio campo de estudio para micólogos y conservacionistas en áreas protegidas por ser uno de los grupos con mayor diversidad biológica de la tierra (Baldrian, 2004). Pertenecen al Reino Fungi y, a diferencia de las plantas autótrofas fotosintéticas, son organismos heterótrofos que obtienen sus nutrientes por absorción, como resultado de la degradación de compuestos complejos liberados por el medio. Están compuestos por filamentos alargados denominados hifas y por cuerpos fructíferos que, a través de sus esporas, contribuyen a su reproducción (Kuhar et al., 2013). Actualmente, se estima que existen entre 2,2 y 3,8 millones de especies, de las cuales apenas se conoce un $8 \%$ de las aceptadas (Hawksworth et al., 2017).

A través del tiempo, los hongos han demostrado tener altos niveles de adaptación ecológica que les ha permitido progresar en la mayoría de los hábitats terrestres (Orozco-Mosqueda et al., 2015). En los ciclos de la naturaleza su actuación cumple una función transcendental, debido a que además de contribuir al desarrollo de plantas hospedadoras y al sistema de alimentación de algunos animales (Cuevas Moreno, 2016) desempeñan un papel esencial en la dinámica de los ecosistemas, contribuyendo a su desarrollo, estabilidad y funcionamiento (Cepero García et al., 2012); además, favorecen la degradación de materiales muertos que deben ser eliminados para la continuidad de la vida (Ruiz Herrera, 2001) y permiten determinar la biodiversidad, tanto en la superficie como en el interior del suelo.

No obstante, su hospedaje no siempre ha cumplido una función de esta naturaleza; en algunos casos, se han convertido en agentes nocivos que atentan contra sustratos que desean ser preservados (Cuevas Moreno, 2016; Ruiz Herrera, 2001). Ejemplo de esto son los hongos destructores de madera asentados en zonas de conservación.
En República Dominicana, mediante la Ley Sectorial de Áreas Protegidas 202-04 y el Decreto Presidencial 571-09, se ha protegido diversas áreas que constituyen el patrimonio cultural y natural del país, como parques nacionales, monumentos naturales y refugios de vida silvestre que, debido a la gran cantidad de árboles que los componen, se han convertido en zonas hospedadoras de especies macro y micro fúngicas, que tienden a fijarse en la madera de sus árboles (Congreso Nacional, 2009).

Desde el punto de vista ecológico, la madera ha sido considerada como el principal material renovable en la Tierra. Está compuesta esencialmente de celulosa, hemicelulosas y lignina (Schmidt, 2007), macromoléculas que dan forma a la pared celular vegetal. Debido a la acción de algunas especies de hongos, estas macromoléculas han sido degradadas en una gran cantidad de árboles, alterando las propiedades fisicoquímicas del tronco (Martínez et al., 2005). Entre estos organismos se encuentran los Basidiomycotas, un filo de reproducción sexual o asexual que se caracteriza por contener una estructura denominada basidio, donde se forman las basidiósporas durante el proceso de meiosis (Madigan et al., 2015). Generalmente, los hongos pertenecientes a este filo son descomponedores de madera en sustratos de árboles vivos o muertos (Orozco-Mosqueda et al., 2015). Una de las especies identificadas en este tipo de degradación es Trametes hirsuta (Wulfen) Lloyd 1924, un hongo de amplia distribución de la familia Polyporaceae que causa pudrición blanca en madera (Cepero García et al., 2012; Milenković et al., 2018).

En la región del Caribe se ha registrado unas 11,268 especies de hongos distribuidas entre las Islas Bahamas, las Antillas Menores y otros países de la región (Minter et al., citado en Perdomo et al., 2018); en las Antillas Mayores hay evidencias de más de 5,000 Basidiomycetes coleccionados, con un $8 \%$ de estos clasificados (Cantrell et al., 2001; Lodge et al., 2002). 
En la actualidad existen pocos proyectos dedicados a la cuantificación e identificación de hongos en la República Dominicana; no obstante, en el año 2006 se inició un primer intento de inventario en el que se identificó 2,809 especies en el país (Minter \& Perdomo, 2006) y, más recientemente, se ha registrado la presencia de hongos patógenos que causan la muerte en algunos mangles (Perdomo et al., 2018).

Sobre hongos Poriales tenemos los estudios de Ciferri (1929). En su obra Micoflora Domingensis citó algunas especies de este orden; posteriormente, Perdomo (2001), con Inventario de hongos poliporáceos en dos áreas protegidas de la República Dominicana; Perdomo (2002), con una investigación sobre los hongos poroides de Sierra de Bahoruco, República Dominicana; Perdomo \& Ryvarden (2002), con la publicación New reports of polypores from the Dominican Republic y Perdomo \& Lodge (2021), con un checklist titulado Porus: Hongos Poriales de la República Dominicana.

Trametes hirsuta es un hongo patógeno oportunista que se asienta en sustratos de árboles vivos y que persiste como un saprobio, aun después de la muerte del árbol (Farr \& Rossman, 2021), provocando una pudrición blanca en la madera del sustrato (Milenković et al., 2018). Fue descrito por Lloyd en 1924. Los cuerpos fructíferos son de color blanque- cino a pardo amarillento, con tendencia a oscurecimiento a través del tiempo. Los carpóforos presentan forma de abanico con una superficie peluda. Su borde es amarillo tornándose a marrón-ocre. Generalmente se aprecian en troncos de árboles durante todo el año (Laessoe, 2000).

El objetivo del estudio es presentar un nuevo récord de la especie Trametes hirsuta (Wulfen) Lloyd en el Refugio de Vida Silvestre Laguna Mallén, en San Pedro de Macorís.

\section{Materiales y métodos}

\section{Área de estudio}

La Laguna Mallén está ubicada en la zona urbana de la provincia San Pedro de Macorís, específicamente en las coordenadas geográficas $18^{\circ} 27^{\prime} 00^{\prime \prime} \mathrm{N}$ y $69^{\circ} 16^{\prime} 00^{\prime \prime} \mathrm{W}$, con una extensión aproximada de $1,41 \mathrm{~km}^{2}$. Es un humedal de agua salobre declarado mediante decreto presidencial como Refugio de Vida Silvestre del país (Congreso Nacional, 2009). Cuenta con una gran variedad de árboles nativos y endémicos de la isla, además de ecosistemas de manglar que sirven de protección y hábitat para la fauna que se asienta en este lugar. La zona de vida se caracteriza por ser un bosque muy húmedo (López Belando \& Mateo Feliz, 2020) (Figura 1). 
Figura 1. Área de estudio

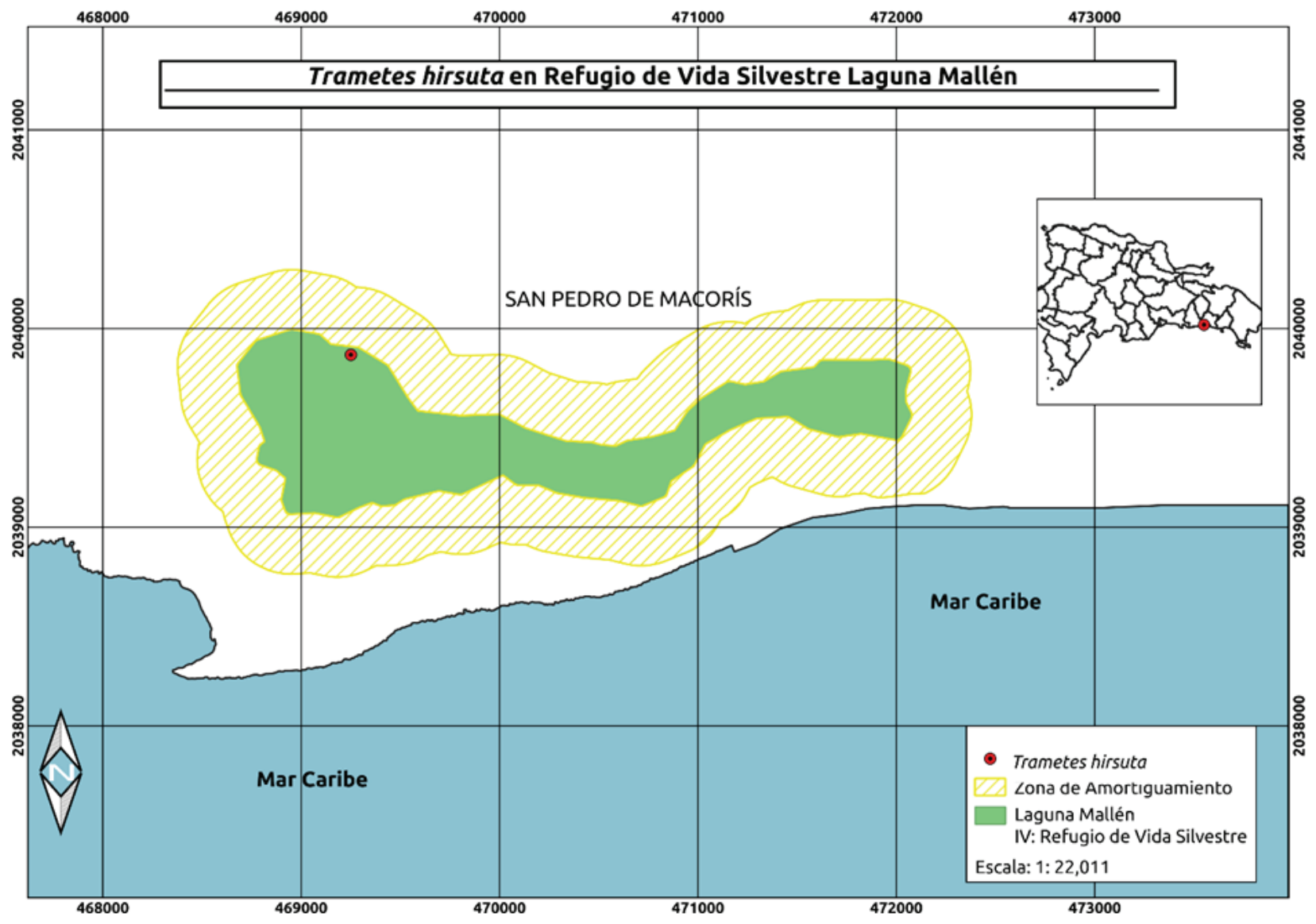

Fuente: (C) Juan Carlos Millord.

\section{Colección de la muestra}

La muestra se recolectó en el mes de marzo del año 2021. El espécimen recolectado se colocó en una bolsa de papel, fue georreferenciado el punto de recolecta y se identificó el árbol hospedero según la morfología de sus órganos (tronco, hojas y frutos) con base a Liogier (2000). Se tomaron anotaciones de las características macroscópicas de la muestra y fotografía in situ. El espécimen fue secado en un deshidratador portátil, a una temperatura de $45^{\circ} \mathrm{C}$ durante 24 horas.

\section{Identificación del espécimen}

Se consideró las características macroscópicas del cuerpo fructífero o carpóforo (forma, superficie y borde), tamaño y color. Se realizó observación de los poros con una lupa (LW Sientific DM Series) y para la observación microscópica (sistema hifal, basidios y esporas) se realizaron secciones a mano del himenio, utilizando $\mathrm{KOH}$; para las observaciones se utilizó un microscopio compuesto con cámara integrada (LW Scientific). Se elaboraron dibujos microscópicos de las estructuras observadas. Para la identificación de la especie nos auxiliamos de un especialista y de la literatura especializada de Gilbertson \& Ryvarden (1987).

\section{Resultados y discusión}

Trametes hirsuta (Wulf.: Fr.) Pil. Atl. Champ. Europe 3: 265, 1939; Polyporus hirsutus Wulf.:Fr., Mycol. 1: 367, 1821 (Figura 2). 
Basidiocarpos: anuales, sésiles, con forma dimidiado, de 41-60 × 15-25 x $1 \mathrm{~mm}$. Superficie del píleo: se caracteriza por ser zonada y concéntricamente sulcada a tomentosa, con una coloración de crema-amarillento a gris, margen del píleo marrón, delgado y entero. Consistencia: va de flexible cuando fresco a dura cuando seco. Superficie de los poros: de color crema-amarillo pálido, los poros con una morfología de redondos a angulares, de 3-4 por $\mathrm{mm}$. Tubos: se caracterizan del mismo color de la superficie de los poros, hasta $2 \mathrm{~mm}$ de grosor. Contexto: doble con la capa superior gris, fibrosa con línea negra que la separa de la capa inferior, hasta $2 \mathrm{~mm}$ de grosor.

Sistema hifal: trimítico, las hifas generativas de pared delgada, hialinas, fibuladas, 2.5-6 $\mu \mathrm{m}$, hifas esqueléticas de pared gruesa, hialinas, no septadas, 2.5-7.5 $\mu \mathrm{m}$, las hifas ligadoras de pared gruesa, hialinas, no septadas, 2.5-5 $\mu \mathrm{m}$. Cistidios: y demás elementos estériles ausentes. Basidios: con forma clavada, 15-20 x 5-7 $\mu \mathrm{m}$. Basidiósporas: cilíndricas, hialinas, lisas, $6-9 \times 2-2.5 \mu \mathrm{m}$.

Tipo de podredumbre: blanca.

Sustrato: sobre tronco de Hura crepitans L. (Euphorbiaceae), un árbol nativo de regiones tropicales de América que a nivel ecológico se caracteriza por contener grandes reservas de agua ante sequías estacionales. Crece hasta 30 metros de altura, contiene corteza lisa, cubierta densamente de espinas y hojas grandes ovaladas (WFO, 2021). Aunque en el país su uso es meramente ornamental, tanto la sabia como las cápsulas que componen las semillas son potencialmente peligrosas o tóxicas (Iannacone $e t$ al., 2014). Actualmente, es una especie no evaluada por la Unión Internacional para la Conservación de la Naturaleza (UICN) y el Ministerio de Medio Ambiente y Recursos Naturales (MIMARENA) del país (Bobadilla-Peñaló y Acosta Martínez, 2020); sin embargo, los estudios de Jiménez-Orozco et al.
(2019) apuntan a que la especie ha sido introducida en la isla.

Distribución altitudinal en la República Dominicana: 750-1,100 msnm (Perdomo, 2001).

Espécimen examinado: provincia San Pedro de Macorís, Refugio de Vida Silvestre Laguna Mallén, $18^{\circ} 26.9640^{\prime} \mathrm{N}, 69^{\circ} 17.4710^{\prime} \mathrm{W}, 6$ msnm., 07-III2021, Mayeli Jaime De Aza, José Guillermo de la Rosa y Héctor Julio Santos Báez (MJDA-1).

Distribución: En República Dominicana se distribuye en: Cordillera Central, Provincia La Vega, Reserva Científica Ébano Verde: El Arroyazo, 6-I-1997, O. P. Perdomo 72; Omar Paino Perdomo \& D. Jean Lodge 52 (JBSD 88163, CFMR); La Sal, 19-I-1999, O. P. Perdomo \& F Padua 488 (JBSD 91003). Provincia La Vega: Jimenoa, 12-II-1997, O. P. Perdomo 77 (JBSD 88147, CFMR); Jarabacoa, en la carretera camino al Salto de Jimenoa, $19^{\circ} 07^{\prime} 39.6^{\prime \prime} \mathrm{N}-70^{\circ} 36^{\prime} 28.4^{\prime \prime} \mathrm{O}$, 600 msnm., 9-XI-2003, O. P. Perdomo, A. Monegro, D. J. Lodge, T. J. Baroni, O. K. Miller \& H. Miller 1495 (JBSD, CFMR). Parque Nacional J. A. Bermúdez: 27-I-1998, O. P. Perdomo 340 (JBSD 88082, CFMR, O). Provincia Puerto Plata: Estero

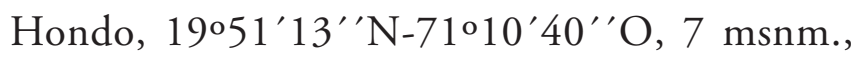
9-I-2002, O. P. Perdomo, N. Marcano \& F. Romano 1175 (JBSD). Provincia Bahoruco: Neyba, La Ceiba, $18^{\circ} 35^{\prime} 03^{\prime \prime N}-71^{\circ} 25^{\prime} 28^{\prime \prime} \mathrm{O}, 814$ msnm., 11-VII2001, O. P. Perdomo, B. Monción \& F. Segura 1047 (JBSD 101831, S). Provincia Pedernales, Parque Nacional Sierra de Bahoruco: en el margen del Hoyo de Pelempito, 7-I-2004, O. P. Perdomo, D. Siri et al., 1620 (JBSD). Provincia Azua: Las Charcas, $18^{\circ} 30^{\prime} 50^{\prime \prime} \mathrm{N}-70^{\circ} 35^{\prime} 21^{\prime \prime} \mathrm{O}, 495 \mathrm{msnm}$., 23-II-2004, O. P. Perdomo, D. de los Santos, T. Montilla \& D. Siri 1629 (JBSD) (Perdomo \& Lodge, 2021). Se distribuye en Norte América (Gilbertson Ryvarden, 1987; Carranza-Morse, 1993), América Central y Europa (Carranza-Morse, 1993). 
Figura 2. Trametes hirsuta
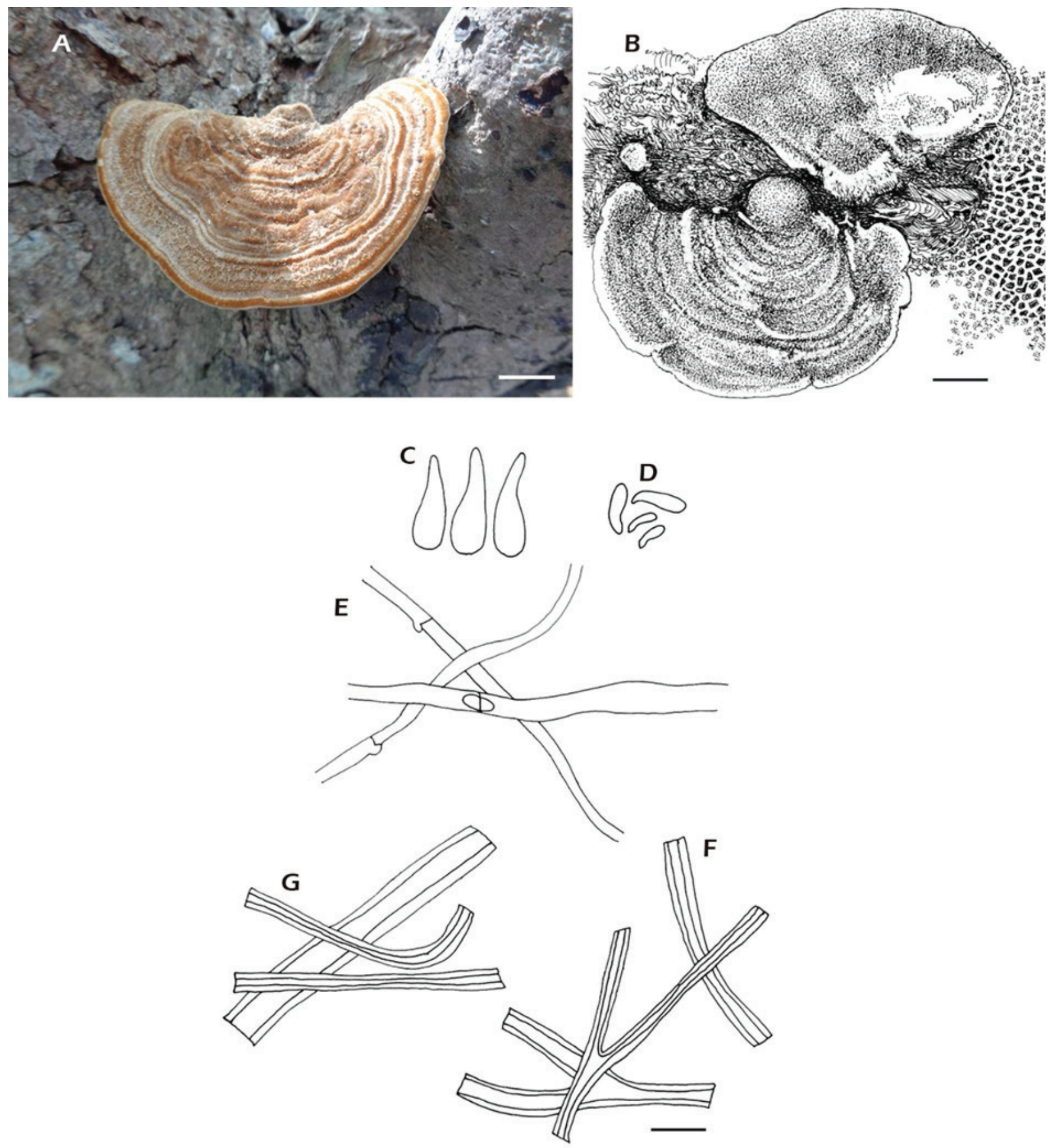

Notas: A. Basidiocarpo in situ. Foto: original de los autores. B. Dibujo basidiocarpo y superficie de los poros (Escalas $=10 \mathrm{~cm}$ ). Ilustración: original Daniel Ortega; C. Basidios D. Basidiósporas E. Hifas generativas F. Hifas ligadoras y G. Hifas esqueléticas (Escalas $10 \mu \mathrm{m}$ ). Dibujos: original de los autores. 


\section{Comentarios}

De acuerdo con Perdomo (2021), especie observada sobre ramas de angiosperma en lugares expuestos y sombreados. La superficie del píleo se caracteriza por tener una coloración gris y un margen de color marrón (Gilbertson \& Ryvarden, 1987). Trametes hirsuta es un patógeno activo, causa pudrición blanca en madera viva de árboles y sobrevive aun después que el árbol muere (Chen et al., 2021; Farr \& Rossman, 2021). Al igual que otras especies del género Trametes, son basidiomicetos cosmopolitas, productores de lacasas (Daâssi et al., 2013; Glazunova et al., 2018) y degradadores de lignina (Anupma \& Tamang, 2020; Knežević et al., 2016; Vasina et al., 2016). Las descripciones macroscópicas y microscópicas coinciden con las de Gilbertson \& Ryvarden (1987) y Perdomo (2001).

Aunque la especie se caracteriza por ser patógena, algunos estudios revelan que posee recursos naturales con un potencial antioxidante y antimicrobiano que se puede utilizar para la elaboración de fármacos (Hasan et al., 2021). Los usos etnobotánicos y su eficaz capacidad para descomponer lignina hacen que la especie juegue un papel importante en la naturaleza, sobre todo en el ciclo natural de los ecosistemas (Chen et al., 2021). La especie puede atacar árboles vivos y permanecer en ellos tras la muerte del árbol, contribuyendo a la descomposición de la madera en su primera etapa (Balaes et al., 2017; Glazunova et al., 2018).

En este estudio se reporta por primera vez en Refugio de Vida Silvestre Laguna Mallén Trametes hirsuta, a una altitud de $6 \mathrm{msnm}$ y por primera vez creciendo sobre Hura crepitans L.

\section{Agradecimientos}

A la Universidad Nacional Pedro Henríquez Ureña (UNPHU), en especial a la profesora Lourdes Rojas, por facilitarnos el uso del laboratorio; a Juan Carlos Millord, por la realización de la cartografía; al ilus- trador Daniel Ortega y a Omar Paíno Perdomo, de la Sociedad Dominicana de Micología, por su asistencia durante el proyecto de investigación. Este trabajo se enmarca dentro del programa de maestría en Biología de la Universidad ISA y el Instituto Superior de Formación Docente Salomé Ureña (ISFODOSU).

\section{Referencias}

Anupma, A. \& Tamang, J. P. (2020). Diversity of filamentous fungi isolated from some amylase and alcohol-producing starters of India. Frontiers in Microbiology, 11(905), 1-16. Doi: https://doi. org/10.3389/fmicb.2020.00905

Balaes, T., Petre, C. V., Ungureanu, C., Mardari, C., \& Tănase, C. (2017). Ligninolytic enzyme system in ecological adaptation of lignicolous macrofungi. Applied Ecology and Environmental Research, 15(1), 207-224. Doi: https:// doi.org/10.15666/aeer/1501_207224

Baldrian, P. (2004). Increase of laccase activity during interspecific interactions of white-rot fungi. FEMS Microbiology Ecology, 50(3), 245-253. Doi: https://doi.org/10.1016/j. femsec.2004.07.005

Bobadilla-Peñaló, E. M. \& Acosta Martínez, L. A. (2020). Diversidad, Estructura y Composición de la Flora Vascular del Campus de la Universidad ISA, Santiago, República Dominicana. Ciencia, Ambiente y Clima, 3(2), 19-36. Doi: https://doi.org/10.22206/cac.2020.v3i2. pp19-36

Cantrell, S. A., Lodge, D. J., \& Baroni, T. J. (2001). Basidiomycetes of the Greater Antilles Project. Mycologist, 15(3), 107-112.

Carranza-Morse, J. (1993). Pore Fungi of Costa Rica III. Mycotaxon, 48, 45-57

Cepero García, M. C., Restrepo, S., Franco Molano, A. E., Cárdenas, M. \& Vargas, N. (2012). Biología de Hongos. 1 ra edición. Bogotá: Ediciones Uniandes. 
Chen, C., Li, Q., Fu, R., Wang, J., Deng, G., Chen, X., $\& \mathrm{Lu}, \mathrm{D}$. (2021). Comparative mitochondrial genome analysis reveals intron dynamics and gene rearrangements in two Trametes species. Scientific Reports, 11(1), 1-12. Doi: https://doi. org/10.1038/s41598-021-82040-7

Ciferri, R. (1929). Micoflora domingensis. Lista de hongos hasta la fecha indicados en Santo Domingo. Estación Agronómica de Moca. Serie B. No. 14.

Congreso Nacional. (2009). Decreto-571-09 (1). $p d f$. Recuperado de: http://ambiente.gob.do/ files/Decreto-571-09.pdf

Cuevas Moreno, J. A. (2016). Los hongos: héroes y villanos de la prosperidad humana. 17(9), 1-10. Recuperado de: http://www.revista.unam.mx/ vol.17/num9/art69/

Daâssi, D., Zouari-Mechichi, H., Prieto, A., Martínez, M. J., Nasri, M., \& Mechichi, T. (2013). Purification and biochemical characterization of a new alkali-stable laccase from Trametes sp. Isolated in Tunisia: Role of the enzyme in olive mill waste water treatment. World Journal of Microbiology and Biotechnology, 29(11), 2145-2155. Doi: https://doi. org/10.1007/s11274-013-1380-7

Farr, D. F. \& Rossman, A. Y. (2021). Fungal Databases, US National Fungus Collections, ARS, USDA. Recuperado de: https://nt.ars-grin. gov/fungaldatabases/

Gilbertson R. L. \& L. Ryvarden (1987). Nort American polypores. Vol. 2. Oslo: Fungiflora.

Glazunova, O. A., Shakhova, N. V., Psurtseva, N. V., Moiseenko, K. V., Kleimenov, S. Y., \& Fedorova, T. V. (2018). White-rot basidiomycetes Junghubnia nitida and Steccherinum bourdotii: Oxidative potential and laccase properties in comparison with Trametes hirsuta and Coriolopsis caperata. PLoS ONE, 13(6), 1-22. Doi: https://doi.org/10.1371/journal.pone. 0197667

Hasan, A., Aslan, A., Akata, I., Gunal, S., Bal, C., \& Baba,H.(2021).Phenoliccontentandbiological activities of Trametes hirsuta. Fresenius Environmental Bulletin, 30(4), 4130-4135. Recuperado de: https://www.researchgate.net/profile/ Hasan-Akguel/publication/350978439_ PHENOLIC_CONTENT_AND_ B I O L O GICAL_ACTIVITIES OF_ TRAMETES_HIRS UTA/ links/607dc238907dcf667baf37d3/ PHENOLIC-CONTENT-AND-BIOLOGICAL-ACTIVITIES-OF-TRAMETES-HIRSUTA.pdf

Hawksworth, D., Gardens, R. B. \& Lücking, R. (2017). Fungal Diversity Revisited: 2.2 to 3.8 Million Species. Microbiol Spectrum, 5(4), 79-95. Doi: https://doi.org/10.1128/microbiolspec.funk-0052-2016

Iannacone, J. A., Ayala, H., Alvariño, L., Paredes Espinal, C., Villegas, W., Alomia, J., Santos, S., Nolazco, N. \& Cruces, L. (2014). Riesgo ecotoxicológico acuático y terrestre del bioplaguicida catahua, Hura crepitans (Euphorbiaceae). Revista de Toxicología, 31(1), 50-62.

Jiménez-Orozco, C., Lebrón-Liriano, B. V., Fernández-Gutiérrez, R., Urbáez, R. \& Guerrero, Ángela. (2019). Caracterización de la flora epífita vascular del Parque Iberoamérica, Santo Domingo, República Dominicana. Ciencia, Ambiente y Clima, 2(1), 23-33. Doi: https:// doi.org/10.22206/cac.2019.v2i1.pp23-33

Knežević, A., Stajić, M., Jovanović, V. M., Kovačević, V., Ćilerdžić, J., Milovanović, I. \& Vukojević, J. (2016). Induction of wheat straw delignification by Trametes species. Scientific Reports, 6(26529), 1-12. Doi: https://doi. org/10.1038/srep26529

Kuhar, F., Castigli, V. \& Papinutti, L. (2013). Reino Fungi: morfologías y estructuras de los hongos. Revista Boletín Biológica, 28(7). https://ri.conicet.gov.ar/bitstream/handle/11336/7765/ CONICET_Digital_Nro.4632_A.pdf Laessoe, T. (2000). ManualesdeIdentificacion-Hongos. Barcelona: Ediciones Omega, S.A. Recuperado de: https://b-ok.lat/book/5877773/0d8e89 
Liogier, A. (2000). Diccionario Botánico de nombres vulgares de La Española. (2a edición). Santo Domingo: Jardín Botánico Nacional.

Lodge, D. J., Baroni, T. J., \& Cantrel, S. A. (2002). Basidiomycetes of the Greater Antilles Project. Tropical Micology, 1, 45-60.

López Belando, A. J. \& Mateo Feliz, J. M. (2020). Guía Ecoturistica del Sistema Nacional de Areas Protegidas. República Dominicana. (1 ra Edición). Santo Domingo: Editora Búho.

Madigan, M., Martinko, J., Bender, K., Buckley, D., \& Stahl, D. (2015). Brock. Biología de los microorganismos (14 ava edición). Madrid: Pearson educación, S.A.

Martínez, Á. T., Speranza, M., Ruiz-Dueñas, F., Ferreira, P., Camarero, S., Guillén, F., Martínez, M., Gutiérrez, A., \& Del Río, J. (2005). Biodegradation of lignocellu-losics: microbial, chemical, and enzymatic aspects of the fungal attack of lignin Lignocellulosic materials. Microbiologia Internacional, 8(3), 195-204. Recuperado de: https://www.scopus.com/inward/ record.url?eid=2-s2.0-24944461038\&partne$\mathrm{rID}=10 \& \mathrm{rel}=\mathrm{R} 3.0 .0$

Milenković, I., Tomšovský, M., Karadžić, D. \& Veselinović, M. (2018). Decline of Paulownia tomentosa caused by Trametes hirsuta in Serbia. Forest Pathology, 48(4), 1-4. Doi: https://doi. org/10.1111/efp.12438

Minter, D. W. \& Perdomo, O. P. (2006). Hongos de la Repuiblica Dominicana-Fungi of the Dominican Republic. Recuperado de: http://www. cybertruffle.org.uk/dorefung/index.htm.

Orozco-Mosqueda, M. del C., Valencia-Cantero, E., López-Albarrán, P., Martínez-Pacheco, M. \& Velázquez-Becerra, C. (2015). La bacteria Arthrobacter agilis UMCV2 y diversas aminas inhiben el crecimiento in vitro de hongos destructores de madera. Revista Argentina de Microbiología, 47(3), 219-228. Doi: https:// doi.org/10.1016/j.ram.2015.06.005
Perdomo, O. P. (2001). Inventario de hongos poliporáceos en dos áreas protegidas de la República Dominicana (Tesis de maestría). Universidad de Costa Rica, Costa Rica.

Perdomo, O. P. (2002). Hongos poroides de Sierra de Bahoruco: República Dominicana. Estudios sobre Los Hongos Latinoamericanos Nacatepec. Veracruz: Guzmán G. \& G. Mata (eds.).

Perdomo, O. P. \& Lodge, J. (2021). Porus: Hongos Poriales de la República Dominicana. Santo Domingo: Editora Búho.

Perdomo, O. P. \& Ryvarden, L. (August, 2002). New reports of polypores from the Dominican Republic, Greater Antilles. En The 7th International Mycological Congress. IMC7 Book of Abstracts. (pp. 11-17), Oslo. https://doi.org/ 10.1016/S1567-1356(03)00007-2

Perdomo, O. P., Miniño, V., Rodríguez de Francisco, L. \& León, Y. (2018). Cytospora rhizophorae Kohlm. \& E. Kohlm (Valsaceae, Ascomycota) en la República Dominicana. Ciencia, Ambiente y Clima, 1(1), 23-31. Doi: https:// doi.org/10.22206/cac.2018.v1i1.pp23-31

Ruiz Herrera, J. (2001). El asombroso reino de los hongos. Avance y Perspectiva, 20, 275-281. Recuperado de: http://www.biomicel.com/ Interes/Tecnologia/34.pdf

Schmidt, O. (2007). Indoor wood-decay basidiomycetes: damage, causal fungi, physiology, identification and characterization, prevention and control. Mycol Progress, 6, 281. Doi: https:// doi.org/10.1007/s11557-007-0534-0

Vasina, D. V., Pavlov, A. R., \& Koroleva, O. V. (2016). Extracellular proteins of Trametes hirsuta st. 072 induced by copper ions and a lignocellulose substrate. BMC Microbiology, 16(1), 1-14. Doi: https://doi.org/10.1186/ s12866-016-0729-0

Word Flora Online, WFO. (2021). Hura crepitans L. Recuperado de: http://www.worldfloraonline.org/taxon/wfo-0000215711 